\title{
INTRAUTERINE GROWTH RESTRICTION (IUGR) RISK DECISION BASED ON SUPPORT VECTOR MACHINES
}

\author{
Zeynep Zengin ${ }^{1}$, Fikret Gürgen ${ }^{1 *}$, Füsun Varol $^{2}$ \\ ${ }^{1}$ Boğaziçi University, Dept. of Computer Eng. Bebek, 34342 Istanbul/Turkey \\ ${ }^{2}$ Trakya University, Ob/Gyn Dept, Edirne/Turkey \\ \{ zeynep.zengin@okan.edu.tr, gurgen@boun.edu.tr, fvarol@yahoo.com.tr\}
}

\begin{abstract}
This paper studies the risk of intrauterine growth restriction (IUGR) using support vector machines (SVM). A structured and globally optimized SVM system may be preferable procedure in the identification of IUGR fetus at risk. The IUGR risk is estimated in two stages: in the first stage, noninvasive Doppler pulsatility index (PI) and resistance index (RI) of umbilical artery (UA), middle cerebral artery (MCA) and ductus venosus (DV) and amniotic fluid index (AFI) are retrospectively analyzed and the Doppler indices are applied to the SVM system to make a diagnosis decision on the fetal wellbeing as "reactive" or "nonreactive and/or acute fetal distress (AFD)" on the nonstress test (NST) (training data). In the second stage (testing data), the decision is validated by the NST (target value). Experiments are performed on previously collected data. Fortyfour preterm with IUGR and without IUGR pregnancies before 34 weeks gestation are considered.The nonparametric Bayes-risk decision rule, k-nearest neighbor (k-NN), is used for comparison. It is observed that the SVM system is proven to be useful in predicting the expected risk in IUGR cases in this small population study. The PI and RI values of UA, MCA and DV are also effective in distinguishing IUGR at risk.
\end{abstract}

Key words- Intrauterine growth restriction (IUGR), Doppler indices PI and RI, support vector machines (SVM), k-NN.

\section{INTRODUCTION}

Intrauterin growth restriction (IUGR) is a fetal growth disorder which is associated with fetal hypoxia and increased perinatal morbidity / mortality [1-5]. The comparison of absolute measurements with reference ranges allows the detection of deviations between expected and actual growth. The classification of fetuses by birth weight percentile has also a significant advantage for detection of IUGR who are at risk for adverse health events.

The relationship between fetoplacental hemodynamic characteristics, fetal behavior, amniotic fluid production and fetal heart rate has been greatly extended by advanced ultrasound technology. Placental hemodynamic problems cause fetal growth delay and adaptive organ responses in uterus. Adaptive responses are intended to enhance fetal survival in hypoxic environment. Exhaustion of placental and fetal adaptive potential leads to decompensation of fetoplacental unit. Severe disturbances of fetal growth is a challenge to the many researchers.

The ultimate severe conditions in IUGR are accepted as absent end diastolic flow or reverse flow in umbilical artery (UA) and high values of UA PI values, decreased PI in 
fetal middle cerebral artery (MCA), appearance of retrograde flow pattern in ductus venosus (DV), severe oligohydramnios $(\mathrm{AFI}<5)$, non-reactivity in nonstress test (NST). These typical features may be employed to monitor the IUGR fetuses at risk.

Support vector machines (SVM) has been one of the most promising statistical decision techniques [6-14]. This is proven in various applications [9-13]. SVM offers some advantages in the risk estimation of IUGR fetuses since it uses support vectors to optimize the decision region along the class boundaries. This gives an opportunity to find the risky IUGR cases. The process of risk estimation has two stages: The first stage is feature extraction for risk estimation. Noninvasive UA, MCA and DV Doppler indices (PI and RI) and amniotic fluid index (AFI) are retrospectively analyzed in actual cases and are labeled as 'reactive' and 'nonreactive' and/or acute fetal distress (AFD) fetuses. Reactive class corresponds to fetuses in normal conditions and 'nonreactive and/or AFD' class corresponds to IUGR fetuses at risk associated with hypoxia. So these features are employed in the SVM system to obtain a two-class decision. In the second stage the fast risk decision is supported by a NST tool. As a result the overall system consists of Doppler readings, SVM based decision and NST tests at cascaded levels for screening the risk in IUGR. Moreover we compare the SVM decision to that of k-nearest neighbor (k-NN) as a nonparametric Bayes-risk estimator. This paper is organized as follows: Section 2 summarizes the risk analysis with SVM classifier. The experimental evaluation and the decision results are given and are compared to the results of $\mathrm{k}-\mathrm{NN}$ in Section 3. Section 4 provides a brief discussion on results and conclusion.

\section{SVM-BASED RISK ANALYSIS}

\subsection{Expected and Empirical Classification Risk}

In the ideal two-class classification problem, the expected risk $\mathrm{R}(\mathrm{f})$ is estimated and minimized under the distribution $P(x, y)$ which is assumed to be known by the choice of a function $f: X \rightarrow\{ \pm 1\}$ from the available set of functions $\mathbf{F}$ based on expected risk minimization principle [6-8]. In practical applications, the distribution should be estimated from the limited number of training data pairs $\left(x_{i}, y_{i}\right), i=1, \ldots, m$ (an ill-posed problem) by implementing the emprirical risk minimization $\left(\mathrm{R}_{\mathrm{emp}}(\mathrm{f})\right)$. The $\mathrm{R}_{\mathrm{emp}}(\mathrm{f})$ is the average value of loss over the training set, while $\mathrm{R}(\mathrm{f})$ is the expected value of loss under the true probability measure.

We can not minimize expected risk directly since the distribution $P(x, y)$ is unknown. However, it is possible to estimate Vapnik-Chervonenkis (VC) bound [3] that holds with the probability $(1-\eta)$

$$
\mathrm{R}(\mathrm{f}) \leq \mathrm{R}_{\mathrm{emp}}(\mathrm{f})+\phi(\mathrm{h} / \mathrm{m}, \ln (\eta) / \mathrm{m})
$$

The bound of the test error depends on the empirical risk and the $V C$ dimension, $h$, defined as the largest number of samples that can be separated using the class of functions $f(x)$. The $V C$ dimension represents the complexity of the function class related to the amount of available training data and the confidence term $\phi$ is referred to as "over fitting". In summary, using the structural risk minimization principle, we 
conclude that the minimum expected risk is obtained by using a SVM model with the minimum sum of empirical risk and by selecting a $V C$ confidence.

\subsection{Linear and non-linear SVMs}

In the linear separable vector space case, we find the optimal hyper-plane with the maximum margin between the classes by the solution of a global maximum or optimum for the quadratic optimization problem. The hyper-plane decision function can be written as

$$
f(x)=\operatorname{sgn}\left(\sum_{i=1}^{m} y_{i} \alpha_{i} \cdot\left(x \cdot x_{i}\right)+b\right)
$$

Here the training samples with nonzero $\alpha_{i}$ 's are called "support vectors" (SV) and the decision function is constructed by only these vectors. The fewer number of SVs indicates better generalization capability.

In the non-separable case, the solution is to allow errors of the SVM by introducing positive slack variables $\xi_{i}, i=1, \ldots, m$, then: $y_{i}\left(w \cdot x_{i}+b\right)-\left(1-\xi_{i}\right) \geq 0, i=1, \ldots, m$. If an error occurs, $\sum_{i} \xi_{i}$ becomes an upper bound on the number of training errors. The decision boundary is then determined by minimizing $\|w\|^{2} / 2+C\left(\sum_{i} \xi_{i}\right)^{k}$, where $C$ is a user-defined parameter indicating the degree of penalty to errors. Now, the only difference from the linear separable case is that the $\alpha_{i}$ have an upper bound of $C$, and the support vectors can lie on the margin or inside the margin. The common approach for separation in this case is to map the original input space to a higher dimensional feature space using kernel functions. As the key idea of non-linear SVMs, kernel functions, $K\left(x_{i}, x_{j}\right)=\Phi\left(x_{i}\right) \cdot \Phi\left(x_{j}\right)$, describe the inner products between vectors $x_{i}$ and $x_{j}$. Then the optimization follows the same procedure as the linear SVM independent from the feature space dimension. Including the kernel inner products, the decision boundary is:

$$
f(x)=\operatorname{sgn}\left(\sum_{i=1}^{m} \alpha_{i} y_{i} K\left(x, x_{i}\right)+b\right)
$$

The choice of the kernel function is critical for the success of the SVM classifier.

\subsection{IUGR Risk Decision based on SVM}

The Doppler indices of placental fetal vessels (PI, RI's of UA, MCA, DV vessels) and amniotic fluid index (AFI) are inputted to the supportive system based on SVM to predict preterm IUGR cases at a minimum expected risk ( Figure 1). The SVM distinguishes reactivity and nonreactivity and/or fetal distress findings as an indication of placental function/dysfunction at the first stage. In the second stage, SVM findings are validated by NST value (reactivity,nonreactivity and/or fetal distress). 


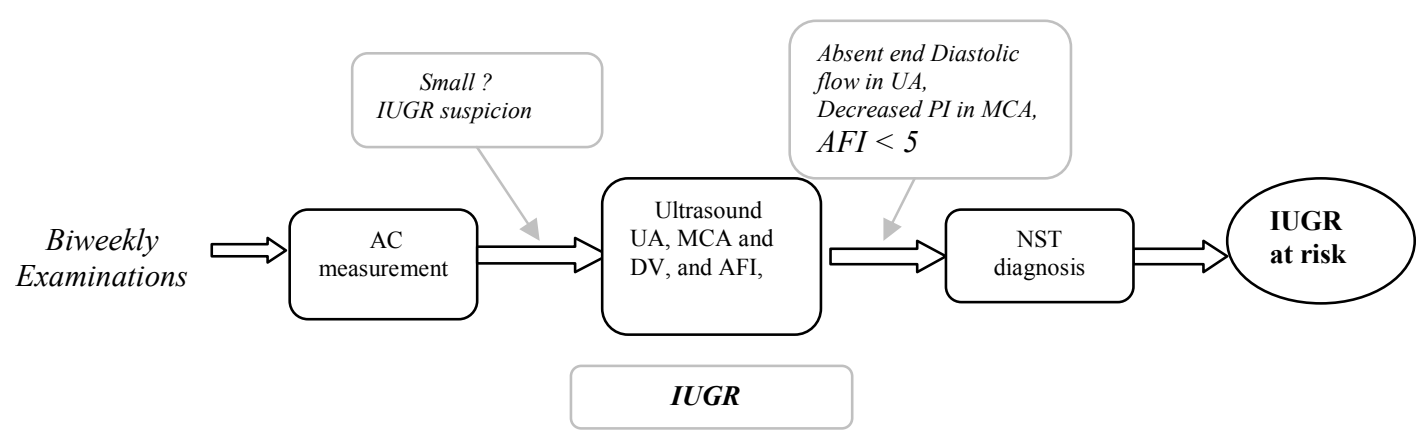

Figure 1: A Flowchart for IUGR fetuses at risk

The advantageous properties of the SVM model in the IUGR application are to give a single solution characterized by global minimum optimized functions and not to rely so heavily on heuristics. Finally it's flexible structure obtains a minimum expected risk by $\mathrm{VC}$ bound. So the risk limits in IUGR may be adjusted by a $\mathrm{C}$ parameter choice. Once the related support vectors are derived from IUGR input space, a single clear decision on risky cases can be made.
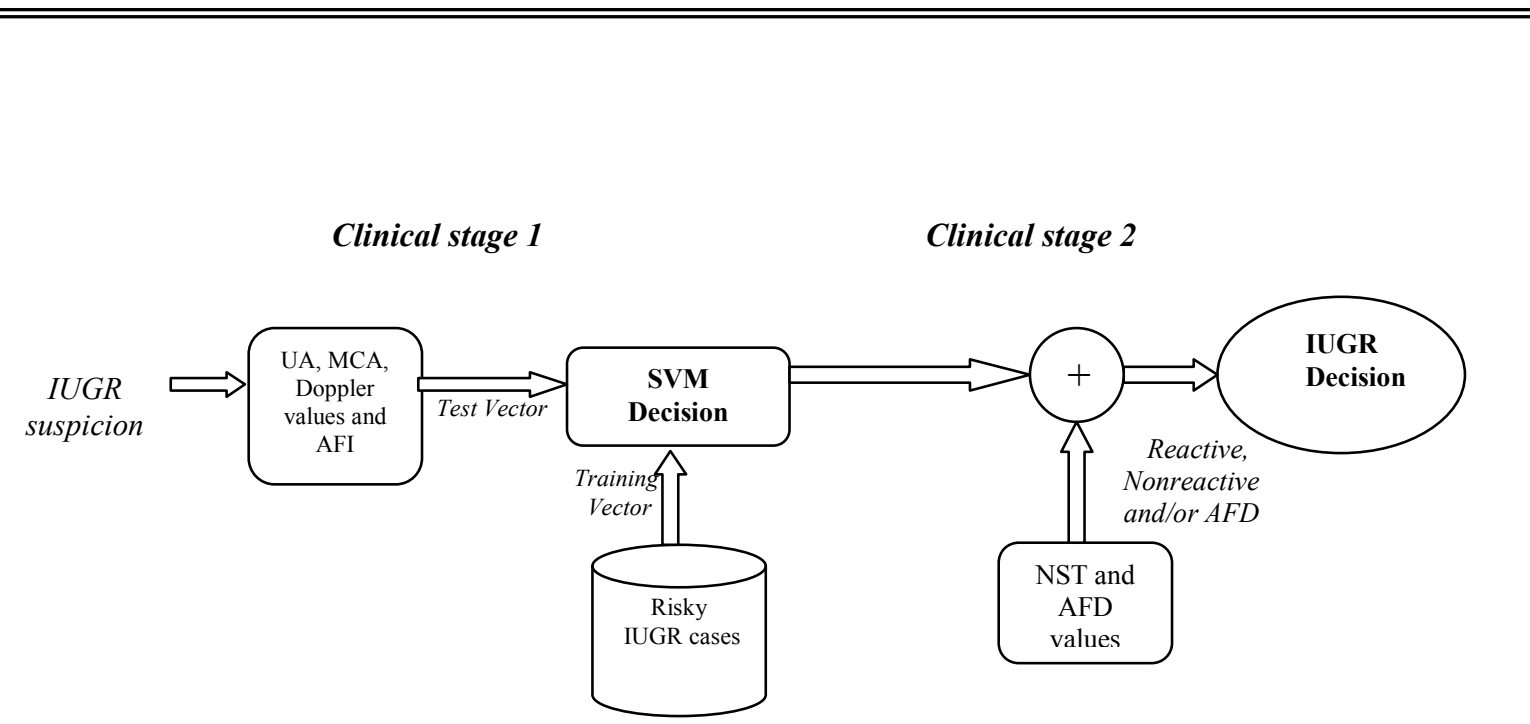

Figure 2: IUGR Decision at Minimum Expected Risk

Noninvasive measurements of PI, RI's and AFI's to make dependable decision in a short time interval is a necessity and an instant minimum expected risk estimation is also vital point in IUGR management . In this aspect, a fast noninvasively obtained feature set and globally minimized flexible SVM system appears to be good choice. 


\section{RESULTS}

Fortyfour preterm pregnancies $(<34$ weeks) with IUGR and without IUGR were analyzed retrospectively [2]. This study has been approved by ethical committe (Trakya University Medical School Ethics Committee - protocol no: TUTFEK-2004/057, protocol date: 13/05/2004. Group 1 includes 18 pregnancies with IUGR $(\% 40.1)$ and group 2 has 26 pregnancies without IUGR (\%59.9) (Table 1). Two classes defined as 'reactive' and 'nonreactive and/or AFD'.

Table 1: Features of 44 pregnancies with IUGR and without IUGR

\begin{tabular}{|l|l|l|l|}
\hline & $\begin{array}{l}\text { Group 1 with } \\
\text { IUGR }(n=18)\end{array}$ & $\begin{array}{l}\text { Group 2 without } \\
\text { IUGR }(n=26)\end{array}$ & overall $(n=44)$ \\
\hline Age & $25,67 \pm 4,3$ & $27,35 \pm 5,9$ & $26,7 \pm 5,3$ \\
\hline UA - PI & $1,41 \pm 0,08$ & $1,08 \pm 0,08$ & $1,60 \pm 0,35$ \\
\hline UA - RI & $0,77 \pm 0,35$ & $0,70 \pm 0,28$ & $0,80 \pm 0,08$ \\
\hline MCA - PI & $1,49 \pm 0,38$ & $1,67 \pm 0,5$ & $1,22 \pm 0,32$ \\
\hline MCA - RI & $0,78 \pm 0,34$ & $0,82 \pm 0,35$ & $0,73 \pm 0,12$ \\
\hline DV - PI & $0,77 \pm 0,13$ & $0,63 \pm 0,12$ & $0,69 \pm 0,24$ \\
\hline DV - RI & $0,58 \pm 0,28$ & $0,50 \pm 0,17$ & $0,53 \pm 0,14$ \\
\hline AFI & $4,56 \pm 0,76$ & $6,62 \pm 0,51$ & $5,77 \pm 3,8$ \\
\hline
\end{tabular}

Doppler PIs and RIs of UA, MCA, DV and AFI values were the data chosen to demonstrate an ability for SVM to extract information. The SVMs, besides their Lagrangian formulation, can differ in two aspects : (i) coefficient C controlled capacities (ii) the anisotropic radial basis in the Gaussian kernel transformation controlled classifier functions.

All data from 44 preterm pregnancies are divided into two halves: training and test data. Thirtyfour values are reserved for training and the rest (a total of 10 values is constructed by a combination of 6 reactive and 4 nonreactive and/or AFD cases at each experiment) is used for testing. The experiments are repeated 10 times for unseen test values and the average performance is computed.

Different cases are considered: Locally linear classifier functions with capacity fixed at $\mathrm{C}$ value is employed. A linear combination of predictors weighted with various coefficients approximates the discriminating rule in this case. A more detailed picture is seen with the increasing capacity of classifier functions. Normal and risky areas become localized. By decreasing the radial basis the SVM will try each case and the complexity will also be increased. For large $C$ values the SVM only localized to one cluster of nonreactive cases. The area outside this cluster is associated with approximately equally high score values. The area outside this clusteris associated with approximately equally high score values. The SVM manages to learn non-reactive cases in even a small size population. This result is obtained by training (Figure 3). Figure 4 demonstrates sample distribution and the support vectors in PI- UA and PI-MCA space. The reactive and non-reactive classes are described with 'star' and 'circle'. The squared star and circle show the support vectors computed by training samples. The dark star and circle show reactive and non-reactive samples of the experiments. 


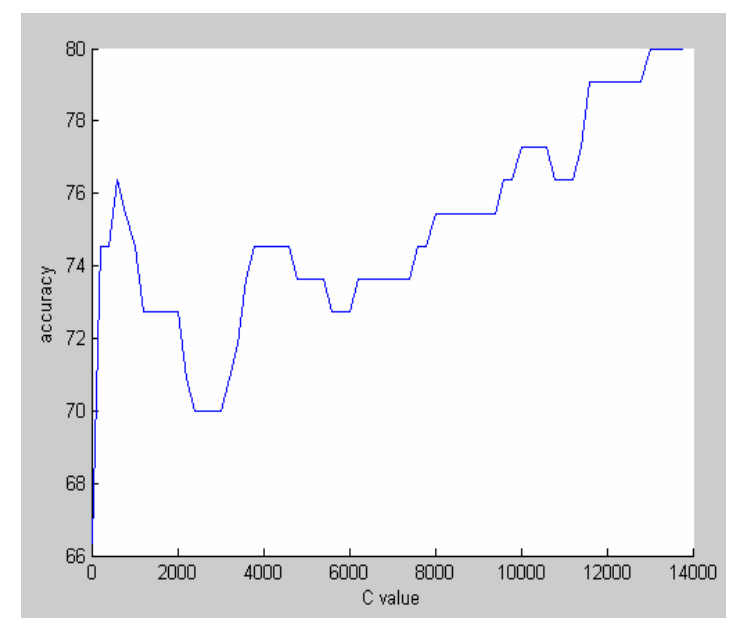

Figure 3: The relationship between performance and $\mathrm{C}$ value

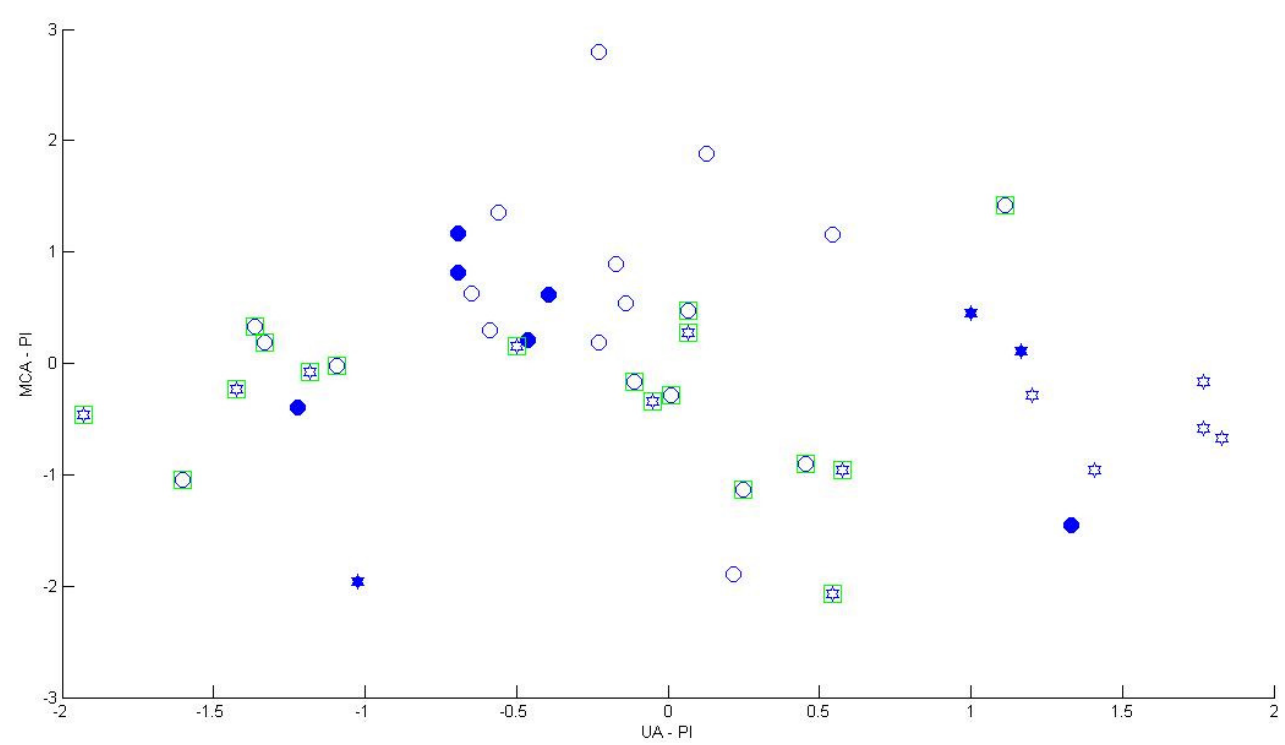

Figure 4: The distribution of samples and support vectors of PI-UA and PI-MCA space. The reactive and non-reactive classes are denoted by 'star' and 'circle'. The 'squared star' and 'squared circle' show the support vectors. The 'dark star' and 'dark circle' show reactive and non-reactive test samples.

The SVM decision results with parameters $(C=13000, \gamma=0.03)$ in small population experiments are represented in Table 2. Various combinations of input features ( y:yes and n:no) in the classification task and accuracies are examined: First,it is observed that SVM becomes an effective tool in management of the IUGR. Second, PI feature is effective in SVM experiment and UA-PI, MCA-PI and RI measurements gives the best accuracy of the system. Non-metric,bi-valued AFI is also supporting evidence of 
IUGR. Finally The PI values obtained from all vessels have separating ability IUGR at risk.

Table 2: Classification performances for various combinations of PIs and RI s of UA,

\begin{tabular}{|c|c|c|c|c|c|c|c|}
\multicolumn{10}{|c|}{ Experiment } & $\mathbf{1}$ & $\mathbf{2}$ & 3 & $\mathbf{4}$ & 5 & $\mathbf{6}$ \\
\hline \multirow{2}{*}{ UA } & $\mathbf{P I}$ & $\mathrm{y}$ & $\mathbf{y}$ & $\mathrm{y}$ & $\mathrm{Y}$ & $\mathrm{y}$ & $\mathrm{y}$ \\
\cline { 2 - 8 } & $\mathbf{R I}$ & $\mathrm{y}$ & $\mathbf{y}$ & $\mathrm{y}$ & $\mathrm{N}$ & $\mathrm{n}$ & $\mathrm{n}$ \\
\hline \multirow{2}{*}{ MCA } & $\mathbf{P I}$ & $\mathrm{y}$ & $\mathbf{y}$ & $\mathrm{n}$ & $\mathrm{Y}$ & $\mathrm{y}$ & $\mathrm{n}$ \\
\cline { 2 - 8 } & $\mathbf{R I}$ & $\mathrm{y}$ & $\mathbf{y}$ & $\mathrm{n}$ & $\mathrm{N}$ & $\mathrm{n}$ & $\mathrm{n}$ \\
\hline \multirow{2}{*}{ DV } & $\mathbf{P I}$ & $\mathrm{y}$ & $\mathbf{n}$ & $\mathrm{n}$ & $\mathrm{Y}$ & $\mathrm{n}$ & $\mathrm{n}$ \\
\cline { 2 - 9 } & $\mathbf{R I}$ & $\mathrm{y}$ & $\mathbf{n}$ & $\mathrm{n}$ & $\mathrm{N}$ & $\mathrm{n}$ & $\mathrm{n}$ \\
\hline \multirow{2}{*}{$\begin{array}{c}\text { Accuracy } \\
\text { (\%) }\end{array}$} & 73 & $\mathbf{8 1}$ & 69 & 77 & 75 & 74 \\
\hline
\end{tabular}

The accuracy,sensitivity,specificity and positive predictive value (PPV) for selected features in SVM system are shown in Table 3. It is observed that PI values in UA,MCA and DV introduce a good and balanced sensitivity and specificity results in the experiments. Addition of RI to the PI UA and MCA has positive effect on accuracy, specificity and PPV value however sensitivity drops.

Table 3: The accuracy, sensitivity, specificity and PPV values for the selected features in SVM system

\begin{tabular}{|c|c|c|c|c|}
\hline SVM results & Accuracy (\%) & Sensitivity(\%) & Specificity (\%) & PPV (\%) \\
\hline PI, RI in UA and MCA & 81 & 62.5 & 93.3 & 86.2 \\
\hline PI in UA, MCA and DV & 77 & 70 & 81.7 & 71.8 \\
\hline PI in UA and MCA & 75 & 60 & 85 & 72.7 \\
\hline PI,RI in UA, MCA, DV & 73 & 62.5 & 80 & 67.6 \\
\hline
\end{tabular}

Furthermore Bayes-risk estimation was employed for comparison purposes. Because of limited number of samples nonparametric k-NN decision rule becomes a proper choice. In the clinical situation of 44 pregnancies, $k-N N$ experiments for $k=1$ to $k=7$ are performed with best performance (Table 4). Although k-NN classifier gives comparable performance to SVM system the balanced sensitivity and specificity values are only obtained with the SVM system.

Table 4: The accuracy, sensitivity, specificity and PPV values for the selected features in Bayes-risk estimate through k-NN decision rule.

\begin{tabular}{|c|c|c|c|c|c|}
\hline k-NN decision rule & Accuracy (\%) & Sensitivity(\%) & $\begin{array}{c}\text { Specificity } \\
(\%)\end{array}$ & PPV (\%) & k \\
\hline PI, RI in UA and MCA & 79 & 55 & 95 & 88 & 5 \\
\hline PI in UA, MCA and DV & 75 & 52.5 & 90 & 77.8 & 5 \\
\hline PI in UA and MCA & 76 & 57.5 & 88.3 & 76.7 & 7 \\
\hline PI,RI in UA, MCA, DV & 76 & 60 & 86.7 & 75 & 1 \\
\hline
\end{tabular}




\section{DISCUSSION AND CONCLUSION}

Prognostic role of fetal Doppler velocimetry in growth-restricted fetuses can not be overlooked. The proposed SVM based decision in IUGR is presented. In the first stage Doppler indices of UA, MCA and DV, AFIs and fetal responses in NST are retrospectively analyzed. In the second stage validation of results are done using NST tool. So SVM system is employed for a fast and minimum expected risk estimation of each case.

Although the data given is extracted from a small population, SVM s are still capable of extracting information with $70 \%$ of a sensitivity, $81.7 \%$ of specificity with PI indices of UA, MCA and DVs. The effectiveness of the system is supported by $71.8 \% \mathrm{PPV}$ on diagnosis of hypoxia suspicion. However the inclusion of UA and MCA RIs drops the sensitivity to $62.5 \%$. This causes an increase on the false negativities and diagnosis of more non-reactivities as reactive. The inclusion of RIs causes unbalancing effect. In such high risk pregnancies these supportive computation methods may draw obstetrician's attention to evidences of unexpected hypoxic events and further analysis into IUGR

It is observed that the inclusion of RI improves the accuracy $81 \%$ on diagnosis of IUGR cases. Both indices ,PI and RI employ information on "systolic-end-diastolic peak velocity" in common, but their usage of the data on "time-averaged maximum velocity " and "systolic peak velocity" differ. While "systolic peak velocity" may properly classify risky pregnancies but does not contribute to a balanced improvement of both sensitivities and specificities.

The detection of AFI as being $<5$ makes the most important contribution to these fuzzy Doppler indices. This bi-valued non-metric feature commonly occurs with the disturbance in UA PI ( and PIs in MCA and DV later on) and finally nonreactivities and fetal distress are seen on NST recordings.

The proposed SVM system is compared with k-NN as a nonparametric Bayes-risk estimator. It is found that k-NN also performs reasonably well interms of accuracy, specificity and PPV. However SVM does much better in sensitivity and gives a balanced pair of sensitivity and specificity. It is also observed that SVM system is superior to the other neural structures.

As a conclusion, SVMs give an opportunity to obtain the results not very obvious at first glance and to easily tune with only a few parameters such as risk estimation in IUGR.

SVMs in IUGR management are based on very few restrictive assumptions and can reveal some important features overlooked by many other methods. Therefore they may become an option of choice at risk decision of IUGR. Although a very limited data in IUGR are retrospectively analyzed, the results are meaningful. On emay extend this study with new and large sets of data and alternative formulations of SVMs better suited for processing large data sets.

\section{Authors' Contributions}

FV supervised the data collection and discussions in Ob/Gyn. Area. FG supervised and prepared the manuscript. ZZ carried out the numerical experiments. 


\section{Acknowledgement}

The work presented in this paper has been supported by Boğaziçi University Research Fund (BAP 09A102P project).

\section{REFERENCES}

1. James D.K., Steer P.J., Weiner C.P., Gonik B., High Risk Pregnancy Management Options, Third Edition, Fetal Growth Disorders, Chapter 12 by Ahmet Alexander Baschat, Third Edition, Saunders Elseiver, 240-265, 2006.

2. Balık G. (Supervisor Füsun Varol), Relationship Between Veous Doppler and Perinatal Outcomes in Fetal Growth Restriction, Gynecology and Obstetrics Speciality Thesis, Trakya University, 2005.

3. Campbell S., Warsof S. L., Little D., et al., Routine Ultrasound Screening for the prediction of Gestational Age, Obstet Gynecol, 65, 613-620, 1985.

4. Gürgen Fikret, Önal Emrah, Varol Füsun, IUGR Detection by Ultrasonographic examinations using neural networks, IEEE Eng Medi Biol Mag, 16, No 3, 55-58, May/june, 1997.

5. Gürgen Fikret, Güler Nilgün, Varol Füsun, Antenatal Fetal Risk Assessment Using a NeuroFuzzy Technique, 20, No 6, 165-169, 2001.

6. Moguerza, J. M., Munoz, A. Support Vector Machines with Applications. Statistical Science. 21, 322-226, 2006.

7. Vapnik V., The Nature of Statistical Learning Theory, Springer Verlag, NewYork, NY, 1995.

8. Bartlett P. L., Jordan M. I. and McAuliffe J. D., Convexity, Classification, and Risk Bounds, Journal of the American Statistical Association, 101, 138-156, 2006.

9. Ding C., and Dubchak I., Multi-class protein fold recognition using support vector machines and neural networks. Bioinformatics 17 349.358, 2001.

10. Furey T. S., Cristianini, N., Duffy, N., Bednarski, D., Schummer, M. and Haussler, D. Support vector machine classification and validation of cancer tissue samples using microarray expression data. Bioinformatics 16 906.914, 2000.

11. Hardle W., Moro R. A., Schafer D., Predicting Bankruptcy with Support Vector Machines, SFB 649 2005-009, Humboldt-Universität zu Berlin, Germany, 2005.

12. Filiz Güneş, Nurhan Türker, Fikret Gürgen, "Signal-Noise Support Vector Model of a Microwave Transistor," Intl. Journal of RF and Microwave Computer AidedEngineering, 404-415, July 2007.

13. Lunghi F., Magenes G., Pedrinazzi L. And Signorini M. G., Detection of Fetal Distress through a Support Vector Machine based on Fetal Heart Rate Parameters, Computers in Cardiology, 25, Issue 25-28, 247-250, 2005.

14. Hsu, C. W., Chang C. C., Lin C. J., A Practical Guide to Support Vector Classification, Dept. Of CS and IE, National Taiwan University, Taipei, 2001. 